


RIPS: Revista de Investigaciones Políticas y Sociológicas, 20(1), 2021. ISSN: 2255-5986

https://doi.org/10.15304/rips.20.1.7087

Artículos

\title{
Cohesión familiar y covid-19: los efectos de la pandemia sobre las relaciones familiares entre los jóvenes universitarios madrileños y sus padres
}

Family cohesion and covid-19: the effects of the pandemic on family relationships between university students and their parents in Madrid

\author{
Giuliano Tardivo ${ }^{1}$, Álvaro Suárez-Vergne ${ }^{2}$, Eduardo Díaz Cano ${ }^{3}$ \\ ${ }^{1}$ Universidad Rey Juan Carlos \\ ${ }^{2}$ Universidad Complutense de Madrid \\ ${ }^{3}$ Universidad Rey Juan Carlos
}

Recibido: 07/09/2020; Aceptado: 03/02/2021

\section{Resumen}

El objetivo de esta investigación, que aquí presentamos de forma resumida, no es otro que comprobar qué consecuencias está teniendo la COVID-19 y el confinamiento forzado sobre la cohesión familiar. Nuestras unidades de observación son los jóvenes universitarios madrileños, que estudian en universidades públicas, como la URJC, la UCM y la UAM y que cohabitan con algunos familiares. La recogida de los datos ha tenido lugar durante el primer confinamiento por el COVID (marzo y abril de 2020). Como técnica de recogida de los datos hemos elegido la encuesta online, teniendo en cuenta las dificultades del momento. Al final, han participado en nuestro estudio 305 estudiantes universitarios. Los resultados conseguidos son contradictorios y ambivalentes: una mitad de los encuestados declara que no se han producido cambios, y la otra mitad se divide entre quienes hablan de cambios positivos y quienes de cambios negativos. Podemos hablar de cuatro grupos o categorías de personas distintas: de relaciones intensas, fortalecidas, sin cambios y conflictivas. Concluimos el estudio subrayando la necesidad de llevar a cabo en el futuro estudios cualitativos para profundizar y matizar los resultados conseguidos en la presente investigación, así como la realización de estudios longitudinales, para tener en cuenta si los cambios producidos por la COVID-19 sobre la cohesión familiar son interpretables como pasajeros o permanentes.

Palabras clave: COVID-19; Cohesión familiar; Individualización; Diálogo; Privacidad

\begin{abstract}
The objective of this research, we present here in a summarized form, is none other than to verify what consequences the COVID-19 and forced confinement is having on family cohesion. Our observation units are young students at public universities from Madrid as they are UCM, UAM and URJC. These students live with some of their relatives. The survey was launched while the first COVID-Confinement took place (March and April 2020). We have chosen the online survey as a technique for collecting data, taking into account the difficulties of the moment. In the end, 305 university students took part in our study. The results


are contradictory and ambivalent: one half of the respondents stated that no changes had taken place, and the other half was divided between those who spoke of positive changes and those who spoke of negative changes. We can talk about four different groups or categories of people: intense, strengthened, unchanged and conflictive relationships. We conclude the study by stressing the need to carry out qualitative studies in the future in order to deepen and qualify the results achieved in the present research, as well as to carry out longitudinal studies, in order to take into account whether the changes produced by the COVID-19 on family cohesion are interpretable as temporary or permanent.

Keywords: COVID-19; Family Cohesion; Individualization; Dialog; Privacy

\section{Introducción}

La COVID-19 se está demostrando cada día más un hecho social total, un fenómeno que abarca todas las dimensiones sociales, incluso la familiar (Santoro, 2020, Yi-Ling, 2020). Por otro lado, la Sociología ha tenido un cierto espacio en las reflexiones académicas y periodísticas sobre la crisis de la COVID-19: pensemos en el artículo de Julio Carabaña publicado en El País del 8 de abril de 2020, sobre la necesidad de utilizar muestras representativas para estudiar la extensión del contagio (Carabaña, 2020). Como recuerda Eva Millet (2020), el confinamiento ha determinado que la mayoría de los jóvenes pase las 24 horas diarias con sus padres, una novedad absoluta, teniendo en cuenta la pérdida de influencia que habían tenido los padres en las últimas décadas como agencia de socialización, respecto a las Tablet, a las TIC y a los grupos de pares. Como reconoce el mismo Luhmann (Cadenas, 2015: 38), una parte importante del proceso socializador ocurre fuera del control que puede "ejercer la familia". Hemos pasado en pocos días de la sociedad del cansancio, descrita por Byung Chul Han (2010), que obliga a los padres a acrobacias continuas (Beck y BeckGernsheim, 2003: 185), a la sociedad del aburrimiento, como consecuencia de un parón inesperado, prolongado y repentino, respecto a un "mundo de velocidad y aceleración continua" (Bauman, 2018: 31). Cabe tener en cuenta que, de todas formas, muchos psicólogos sociales aconsejan el aburrimiento para el crecimiento de los adolescentes. Alain Touraine ha hablado de época de vacío y

de no sentido. Por consiguiente, es necesario investigar sobre el hecho de que la familia y la cohesión familiar puedan constituir unos antídotos contra este vacío, emocional y social (Bassets, 2020).

La principal pregunta de investigación a la que queremos contestar, a través del presente trabajo, es la siguiente:

¿Ha variado la percepción que tienen los jóvenes estudiantes universitarios madrileños de las relaciones familiares con sus padres como consecuencia del confinamiento?

Que a su vez se desglosa en cuatro preguntas específicas:

¿Los estudiantes universitarios madrileños tienen la sensación de que ha aumentado la cohesión familiar, en el seno de sus familias, como consecuencia de la pandemia y del confinamiento?

¿Los estudiantes universitarios madrileños han sentido invadido su espacio personal (por sus padres) durante el confinamiento o, al contrario, el hecho de pasar más horas juntos ha determinado un reforzamiento de los lazos afectivos familiares y de la sociabilidad?

¿Han aumentado cualitativa y cuantitativamente las conversaciones familiares? A este respecto, cabe recordar que, según un estudio del CIS de 2014, el 68,4\% de los padres españoles encuestados declaraba que la comunicación con los hijos no constituía un problema. 
¿Las TIC, las pantallas digitales, las redes sociales, han influido en el desarrollo de las relaciones familiares durante el confinamiento? Según la encuesta del CIS de 2014, es decir muy anterior a la aparición de la COVID 19 (CIS, 2014: 14), el uso de videojuegos, televisiones, Tablet y dispositivos móviles, sí que era percibido como un problema, grave o leve, por la mayoría de los padres, y se asociaba a menor diálogo y más conflicto intrafamiliar (CIS, 2014: 19). De hecho, son los jóvenes de entre 16 y 24 años los que tienen de promedio mayores habilidades digitales (INE, 2019: 4). El uso de la red en esta cohorte "es prácticamente universal" (INE, 2019: 5).

El objetivo de este trabajo es averiguar si la percepción de los estudiantes universitarios madrileños, que estudian en universidades públicas y que viven con su familia, respecto a la cohesión familiar se ha reforzado o no con la COVID-19 y la cuarentena.

En síntesis, se trata de comprobar si los estudiantes universitarios madrileños que estudian en universidades públicas perciben que los dos fenómenos, COVID-19 y cohesión familiar, están correlacionados (Martínez Pastor, 2019: 79), aunque somos conscientes del hecho que correlación no implique a la fuerza causalidad (Martínez Pastor, 2019: 80). De todas formas, en nuestro caso no puede haber problemas de causalidad inversa o endogeneidad (Martínez Pastor, 2015: 91).

La variable dependiente, que queremos explicar y analizar, es la percepción de la cohesión familiar, y la independiente o posible variable explicativa sería la cuarentena causada por el virus. Aunque sabemos la importancia que tienen algunos factores, como la renta familiar, la clase social o las dimensiones de la vivienda, porque, entre otras cosas, "la infravivienda va a hacer más difícil la actividad diaria" (Bonilla, 2020), en nuestro estudio podemos tomar en consideración solo algunas de estas variables, por la dificultad de conceptualización y operacionalización que tienen algunos de estos conceptos, como el de clase social. De igual manera habría que profundizar otros factores como la precariedad económica, y los riesgos de despido laboral de los padres (Bonilla, 2020), que inevitablemente terminan por condicionar el humor y las relaciones con los parientes más cercanos. Se trata de cuestiones que dejamos para futuras investigaciones.

Como ya han señalado algunos estudios de reciente publicación (Dirección General de Innovación y Estrategia Social, 2020), las consecuencias más negativas de la COVID-19, a nivel económico, están afectando sobre todo a las familias con hijos a cargo y con rentas inferiores a los 2 mil euros y, más aún a las familias monoparentales. Los hogares, que ya estaban en situación de pobreza o en riesgo de pobreza y exclusión social, son en la actualidad los que más están sufriendo las consecuencias de la pandemia. El mismo teletrabajo se ha implementado muy poco en el caso de los trabajadores precarios, con bajos ingresos (Dirección General de Innovación y Estrategia Social, 2020: 3). Como se dice en el mismo estudio de la Dirección General de Innovación y Estrategia Social (2020: 8), "las mayores caídas en los ingresos se dan en los hogares que ya tenían rentas más bajas antes de la crisis". Y se añade: "esto ahondará en la desventaja de los hogares menos favorecidos".

Si el indicador Arope, que mide el riesgo de pobreza y exclusión social, en el año 2018 se situaba en el 19\% (INE, 2018), es de esperar que suba muy considerablemente a lo largo de 2020. Tampoco nos queremos obsesionar con la búsqueda de correlaciones significativas, porque sabemos que "incluso no hallarlas constituye un descubrimiento valioso" (Martínez Pastor, 2019: 107).

Por otro lado, recordamos que la sociedad española se caracteriza por la cercanía física entre las personas. De hecho, en España el vivir solos tiene históricamente "escaso atractivo" (Meil, 2000: 69). Inevitablemente acostumbrarse a mantener relaciones tan prolongadas en el tiempo con los familiares con los que se convive y solo a distancia con los que no son familiares directos, como está ocurriendo como consecuencia de la pandemia del Coronavirus, está siendo complicado. 
Por lo que concierne a la justificación del trabajo, cabe recordar la actualidad del tema a investigar. Como dice Mariano de Urraco (González Rocío, 2020), vivimos en una sociedad que olvida fácilmente, y lo más probable es que, cuando se acabe la pandemia y volvamos a la normalidad, todo lo que emocionalmente ha tenido una importancia crucial en esta situación excepcional pase a un segundo plano. No descartamos la realización de estudios longitudinales a posteriori para averiguar en el futuro si efectivamente se registra esta tendencia y la mayoría de las personas olvidan pronto la COVID-19 con sus consecuencias sociales y familiares. Por último, cabe señalar que, por el momento, se ha publicado solo "alguna investigación incipiente" (Revilla, 2020) sobre los jóvenes y la COVID-19. Como recuerda Hugo Cadenas (2015: 30), "el estudio de la familia parece estar conceptualmente subdesarrollado". Por consiguiente, se necesitan trabajos teóricos y empíricos nuevos y más completos sobre estos importantes asuntos. A continuación, presentamos las principales hipótesis de nuestro estudio:

Hipótesis 1: Es de suponer que se haya incrementado la cohesión familiar como consecuencia de la COVID-19, o por lo menos su percepción por parte de los jóvenes universitarios madrileños que conviven con sus padres.

Hipótesis 2: Pensamos que los estudiantes universitarios madrileños han podido mantener sus espacios personales y su privacidad, a pesar de la larga convivencia forzada con sus padres.

Hipótesis 3: Hipotetizamos que se ha producido un considerable aumento del diálogo intrafamiliar como consecuencia de la convivencia prolongada durante la cuarentena.

Hipótesis 4: Por último, suponemos que el uso prolongado y excesivo de las TIC ha producido una reducción de los efectos benéficos de la COVID 19 sobre la cohesión familiar.

\section{Marco teórico}

El concepto fundamental de nuestra investigación es el concepto de cohesión familiar. En este apartado intentamos definirlo teóricamente, en el siguiente lo operacionalizamos, con el objetivo de medirlo y relacionarlo con la COVID-19 y la cuarentena. Grandes sociólogos del pasado, y también contemporáneos, han estudiado el tema de la cohesión social y familiar y las consecuencias del individualismo creciente sobre nuestra sociedad. Pensemos en los estudios de Durkheim, quien ya habló a finales del siglo XIX de debilitamiento del sentimiento familiar, como consecuencia de la sociedad del asalariado (Segalen, 2013: 82; Cadenas: 29) y de Parsons y, mucho más recientemente en las reflexiones de Beck (2003) y de Bauman (2018), sobre los procesos de individualización y sobre las relaciones afectivas líquidas que parecen prevalecer en nuestra sociedad contemporánea. Estos cambios que se han producido en las últimas décadas parecen haber roto definitivamente con la idea del matrimonio y de la familia como "una especie de ley natural interiorizada" (Beck y BeckGernsheim, 2003: 49).

Hoy, entre los jóvenes adultos, el índice de entropía familiar (Mielo Vieira y Miret Gamundi, 2010: 84) resulta muy elevado, porque existe una notable complejidad y variedad de estatus. Pensemos también en las reflexiones de Martuccelli y Santiago (2017), quienes ponen el foco de atención en los procesos de desinstitucionalización, no solo familiar. A este respecto, Bauman (2018: 10) ha hablado de procesos de "individualización galopante" y de "riesgos y ansiedades de vivir juntos, y separados, en nuestro mundo líquido" (Bauman, 2018: 16). Y el VIII Informe de la Fundación Foessa (2019: 19) pone el foco en una ciudadanía cada vez más "desconfiada, individualista y 
meritocrática". Mientras que Beck y Beck (2003: 32) definieron la familia como "el cubo de la basura de todos los problemas sociales del mundo que no se pueden resolver de ninguna otra manera".

Una de las mejores definiciones del concepto de cohesión familiar la dio Moos en 1976 (Mora Rojas, 1994: 1), quien definió la cohesión familiar como "el grado en que los miembros de la familia se interesan por ella, se comprometen con ella y se ayudan mutuamente". Otra definición interesante la dieron (Mora Rojas, 1994: 3) Rojas y Tichler: la cohesión familiar se podría relacionar, según estos autores, con "la unidad emocional, intelectual y/o física que los miembros de una familia sienten entre sí". Uno de los grandes problemas en las relaciones afectivas actuales es la ausencia de comunicación (Bauman, 2018: 36). Por eso, queremos averiguar hasta qué punto la cuarentena ha influido en la evolución del diálogo intrafamiliar. No podemos utilizar aquí el paradigma de Rolland (2012) porque es sobre todo preventivo y formativo, y esta pandemia nos ha pillado "desprevenidos" pero, quizás aquí, como en todas las crisis -que llegarán sin saber el cuándo- nos hemos olvidado de prepararnos.

En periodos de crisis la solidaridad intrafamiliar suele aumentar, como ocurrió en España durante la crisis económica de 2008-2013, que fue un periodo caracterizado por el crecimiento de las ayudas económicas intrafamiliares y una menor confianza hacia los bancos y las instituciones, como ha podido averiguar en sus investigaciones Gerardo Meil (2011: 20; Tardivo, 2016: 153), y como ha confirmado, más recientemente, el VIII Informe Foessa (2019: 18), en el que se habla de la creciente desconfianza de los españoles "ante los poderes públicos y sus gestores".

El mismo Meil (2000: 19), así como David Reher (1996) y otros sociólogos de renombre, siguen hablando de un modelo familista prevaleciente en las sociedades del Sur de Europa, aunque tampoco niegan la emergencia de un tipo de parentalidad distinto respecto al pasado, más basado en la afectividad que en la consanguineidad.

La importancia de la familia en España es tal que el 85,4\% de los españoles encuestados por el CIS, en 2014, la consideraba como muy importante y otro 13,3\% como bastante importante (CIS, 2014), mientras que solo el 3,6\% se declaraba insatisfecho con su familia. De hecho, según Meil (2000: 8), el retraso de la emancipación juvenil y la crisis del Estado del Bienestar, dos fenómenos especialmente relevantes en el Sur de Europa, se han visto acompañados de una nueva "solidaridad familiar": si no nos hemos hundido del todo "durante la Gran Recesión ha sido en gran parte por la persistencia de vínculos no utilitaristas, especialmente los familiares, que han sostenido y contenido una buena parte de las consecuencias de la misma en el contexto de un débil Estado del Bienestar" (Fundación Foessa, 2019: 24).

La solidaridad familiar se suele percibir sobre todo en los momentos de crisis, lo cual atestigua que la familia hoy se basa en relaciones electivas, pero, cuando es necesario, vuelve a funcionar también como "una comunidad de necesidad" (Beck y Beck, 2003: 146). Se trata de algo que atañe también a los jóvenes: "la juventud percibe a la familia como una de las dimensiones de su vida que más satisfacción le produce” (Erizalde San Miguel, 2020: 34). De los distintos tipos de solidaridad familiar que habríamos podido analizar, nos centramos sobre todo en la solidaridad afectiva y comunicacional (Meil, 2000: 10), es decir tomamos en consideración solo un aspecto "de la multifacética solidaridad familiar" (Meil, 2000: 10). Además, "el ejercicio de equilibrio cotidiano" que está a la base de la convivencia de muchas familias puede haberse complicado durante la cuarentena, o al contrario, puede haberse simplificado (Beck y Beck, 2003: 174). 


\section{Metodología}

La pandemia del COVID-19 y la cuarentena derivada de la misma han generado una situación que se aleja totalmente de la "normalidad", tal y como la entendíamos. De ahí el interés por estudiar las consecuencias de esta y, al mismo tiempo, la necesidad de adaptar estrategias metodológicas para poder hacerlo con la mayor calidad posible. Para ello hay que tener en cuenta dos cuestiones ligeramente contradictorias, por un lado, las dificultades para contactar con la población objeto de estudio (en este caso los estudiantes que viven con sus padres) debido a la propia cuarentena y, por otro, la apremiante necesidad de obtener información sobre las posibles consecuencias de un fenómeno (la cuarentena) para anticiparse a posibles dinámicas negativas derivadas de las mismas.

Partiendo de estas dos premisas, se emplea una perspectiva cuantitativa, haciendo uso de la encuesta que permite recoger unas "grandes cantidades de datos a un precio relativamente bajo en un corto periodo de tiempo" (García Ferrando y Llopis Goig en García Ferrando, Alvira et al., 2015: 340). Se lleva a cabo una encuesta online que evita "el intrusismo entrevistador" (Callejo Gallego, 2019: 138) siendo además la única opción factible (además de la telefónica) dada las características del confinamiento. La recogida de datos ha tenido lugar durante el primer confinamiento, en los meses de marzo y abril de 2020, después de la declaración del Gobierno de Pedro Sánchez del estado de alarma, del día 14 de marzo. Para conseguir la mayor participación posible se ha utilizado la plataforma Google forms difundiendo la encuesta, a través del método de bola de nieve, entre estudiantes de distintos grados de las Universidades Rey Juan Carlos, Complutense y Autónoma para invitarles a participar. Esto permite obtener información de forma rápida y eficaz solventando las dificultades de movilidad asociadas al confinamiento. Hemos elegido como unidades de observación a jóvenes universitarios por razones como la accesibilidad, la disponibilidad a informar y porque, a nuestro juicio, disponen de más información respecto a estudiantes más jóvenes que cursan estudios no universitarios.

Por consiguiente, la población objeto de estudio son los jóvenes estudiantes universitarios de universidades públicas de la Comunidad de Madrid (URJC, UCM y UAM), que tienen entre 18 y 25 años, y que viven con sus padres. Es necesario resaltar que, además de las dos premisas mencionadas anteriormente (la situación de confinamiento y la necesidad de obtener información sobre las consecuencias de ésta de forma rápida) se une la inexistencia de un marco muestral accesible que recoja la población estudiada. Esto implica que el estudio realizado se enfoque desde una perspectiva exploratoria difundiéndose la muestra a través del método de bola de nieve, llevándose a cabo un muestreo por cuotas (sexo). Los resultados se ponderan buscando la similaridad distributiva de la muestra obtenida para con la población objeto de estudio (en este caso, como no existe un marco muestral de los estudiantes universitarios que viven con sus padres, se toma como referencia para las cuotas por sexo a la población universitaria en general de universidades públicas de la Comunidad de Madrid: 225.338), hecho que, si bien no garantiza la representatividad estadística de los resultados, sí inciden muy positivamente en su calidad constituyendo una buena alternativa cuando se carecen de recursos necesarios o las situaciones (como la actual) impiden otros tipos de muestreo (Szolnoki y Hoffmann, 2013; Arroyo y Finkel, 2019).

Dado que el interés del presente estudio gira en torno a las relaciones familiares se propone a continuación un modelo para la medición de las mismas, basado en dos conceptos, la cohesión 
familiar y el respeto/invasión del espacio personal. Dos conceptos que responden a las preguntas específicas que guían el presente estudio.



Fuente: Elaboración propia.

Con el análisis se pretende contrastar el modelo propuesto, clasificando a la población según la percepción del tipo de cambio (o no cambio) producido en sus relaciones familiares durante el confinamiento y observado si estos influyen en la valoración subjetiva de los encuestados de la mejora de sus relaciones familiares y en las discusiones familiares.

Además de ello se analizará si existen diferencias significativas en estas percepciones según variables sociodemográficas como el sexo y la edad del encuestado, así como el nivel de ingresos del hogar en el que reside, el número de convivientes y los metros cuadrados de la vivienda.

Asimismo, se estudia la relación entre las relaciones familiares y otros factores, expuestos anteriormente como son el ocio intrafamiliar y el uso de TICs.

Somos conscientes de que sería interesante complementar la perspectiva cuantitativa, con una mirada cualitativa que permitiese una mirada más comprensiva y cercana. Además, para averiguar si la COVID-19 tiene efectos reales sobre la cohesión familiar se necesitaría una mirada más profunda al pasado, a las relaciones familiares antes del desarrollo de la pandemia, que no podemos llevar a cabo en este contexto. Asimismo, no se puede olvidar que estamos preguntando sobre las percepciones subjetivas de los jóvenes universitarios a propósito de la cohesión familiar, lo cual puede producir sesgos subjetivos, difíciles de calcular, teniendo en cuenta que las percepciones no siempre coinciden con la realidad y que tampoco se suele registrar una coincidencia total entre las percepciones subjetivas de los distintos actores (Martínez Pastor, 2019: 21).

No obstante, como se ha mencionado anteriormente la situación en la que se lleva el trabajo de campo de esta investigación (en pleno confinamiento) ha obligado a los investigadores a adaptar la misma a los recursos disponibles. Por ello, pese a estas limitaciones, se considera que la estrategia metodológica empleada arroja resultados de utilidad para la comprensión cómo está afectando la cuarentena a las relaciones familiares y sus posibles consecuencias, información que sin duda deberá ser contrastada en el futuro con mayor profundidad siendo interesante completar estos 
datos con otros más cualitativos que permitan examinar de cerca el fenómeno explorado en el presente artículo.

\section{Análisis y Resultados}

En total se obtiene una muestra de 305 encuestados: todos los que han participado están estudiando en una de las tres grandes universidades públicas madrileñas y tienen una edad entre los 18 y 25 años, siendo la edad media de 21 años. Además, el muestreo se pondera por cuotas de sexo (46\% hombres, $54 \%$ mujeres), suficiente para cumplir con las ratios necesarias de respuesta para los análisis planteados (Cea D’Ancona, 2002). Para caracterizar la muestra también se tienen en cuenta variables relacionados con el hogar y con la unidad familiar, como los ingresos mensuales del hogar (2.139 euros de media), el total de personas con las que se convive (4 personas de media) y los metros cuadrados de la vivienda (110 metros cuadrados de media).

Cabe mencionar que no se han encontrado diferencias significativas ni por las características del encuestado (sexo y edad) ni por las características de su unidad familiar y su vivienda (nivel de ingresos, total de personas con las que convive y metros cuadrados de la vivienda) por lo que los resultados se exponen sin segmentar según estas variables.

Cuando se pregunta a los estudiantes universitarios que viven con sus padres si la relación con su familia ha cambiado durante la cuarentena, puede observase que más de la mitad de ellos, un $57 \%$, no declaran cambios significativos. Los encuestados que si declaran que sus relaciones han variado, se distribuyen en porcentajes similares entre una mejora (un 25\%) y un empeoramiento $(18 \%)$ de las relaciones familiares.

Gráfico 1. ¿Las relaciones con tu familia han cambiado durante la cuarentena?

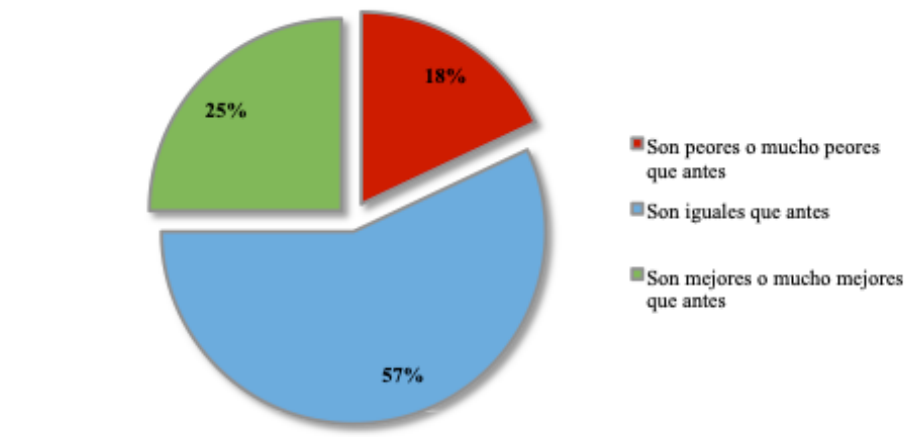

¿Las relaciones con tu familia han cambiado durante la cuarentena?

$\mathrm{N}=305$

Fuente: Elaboración propia.

Este resultado hace necesario examinar las relaciones familiares en mayor profundidad puesto que parece contradecir la idea inicial de que la cohesión familiar ha aumentado durante la cuarentena en términos generales.

Para ello se centra el análisis en las preguntas concretas enfocadas a medir cohesión familiar y la posible invasión del espacio personal. Se lleva a cabo un análisis factorial exploratorio de componentes principales, con las preguntas expuestas en la tabla 1 con objeto de contrastar si realmente esta se puede agrupar en los conceptos latentes operacionalizados anteriormente. 
Se obtiene un modelo de dos componentes que se corresponde con el planteamiento teórico propuesto. Se logra explicar un 69\% de la varianza total, pudiendo considerarse que el constructo creado sintetiza de forma adecuada las variables seleccionadas (Cea D'Ancona, 2002) y se obtiene índice KMO de 0,677, lo que indica que el análisis es pertinente (Kaiser, 1974), lo que se considera un resultado aceptable de cara a aceptar el modelo propuesto. En la tabla 2 se expone la matriz de componentes rotada (rotación Varimax).

Tabla 2. Matriz de componente rotada

\begin{tabular}{|c|c|c|}
\hline & $\begin{array}{l}\text { 1: Incremento } \\
\text { Cohesión } \\
\text { familiar }\end{array}$ & $\begin{array}{l}\text { 2: Invasión } \\
\text { espacio personal }\end{array}$ \\
\hline $\begin{array}{l}\text { La relación con mis padres se ha } \\
\text { fortalecido }\end{array}$ & 0,773 & $-0,370$ \\
\hline $\begin{array}{l}\text { He hablado de temas intimos con mis } \\
\text { padres en mayor medida que antes }\end{array}$ & 0,836 & 0,092 \\
\hline Me siento más unido a mis padres ahora & 0,877 & $-0,242$ \\
\hline $\begin{array}{l}\text { Cada miembro de la familia ha podido } \\
\text { tener su espacio personal }{ }^{*}\end{array}$ & 0,033 & 0,885 \\
\hline Me he sentido agobiado por mis padres & $-0,274$ & 0,558 \\
\hline \multicolumn{3}{|c|}{ Matriz de componente rotada } \\
\hline \multicolumn{3}{|c|}{ * Codificación inversa } \\
\hline
\end{tabular}

Observando los coeficientes factoriales queda patente como las variables incluidas en el análisis se agrupan en torno a dos grandes dimensiones. Los coeficientes factoriales, cuyos valores oscilan entre 1 y -1, nos indican el grado de relación entre cada variable con cada componente. Así puede decirse que con el componente 1 se mide el -Incremento de la cohesión familiar- ${ }^{1}$ puesto que en este saturan una serie de variables que señalan el incremento (o no incremento) de cuestiones estrechamente relacionadas con dicho concepto (se ha fortalecido la relación con los padres, se ha hablado más de temas íntimos, se siente más unido a los padres) mientras que el segundo mide la Invasión del espacio personal-, saturando en las variables "me he sentido agobiado por mis padres" y "Cada miembro de la familia ha podido tener su espacio personal".

Pese a que a priori pudiese parecer que ambos conceptos están estrechamente relacionados, no se obtiene una correlación significativa entre ambos cuando se ejecutan métodos de rotación oblicuos (como la rotación Promax), por lo que se considera adecuada el procedimiento de rotación Varimax. Esto puede explicarse por el motivo de que ciertos "roces" derivados de la estrecha convivencia sean inevitables, y por tanto, sea difícil mantener al completo el espacio personal (al menos en comparación con antes de la cuarentena) lo que no implica que la cohesión familiar pueda mejorar.

Habiendo observado que las relaciones familiares pueden definirse en torno a dos grandes ejes (incremento de la cohesión familiar vs no incremento de la cohesión familiar, invasión espacio personal vs respeto del espacio personal) se procede a clasificar a la población según dichos 
ejes para observar que tipos de variaciones en las relaciones familiares se han dado durante la cuarentena. Para ello se hace uso del análisis clúster K-Means empleando como variables los componentes previamente extraídos mediante el análisis factorial ("Incremento de la Cohesión familiar", "Invasión del espacio personal"). Téngase en cuenta que las unidades de medición de las variables resultado de un análisis factorial son puntuaciones factoriales cuyo valor indica la posición de cada caso sobre la variable creada, pudiendo estas puntuaciones tomar valores positivos o negativos que oscilan (habitualmente) entre 2,5 y -2,5 (valores fuera de este intervalo podrían considerarse como casos atípicos) (Cea D’Ancona, 2002). De manera que valores positivos elevados en un componente, por ejemplo "Incremento cohesión familiar" indican que en ese caso se ha producido un incremento de la percepción positiva respecto a la cohesión familiar, mientras que valores negativos indicarían que no se ha producido un incremento en dicha cohesión. Esto puede hacer que la interpretación de las medias de dichos valores sea a veces confusa. No obstante, dado que en este caso lo que interesa es observar cómo se clasifica la población sobre la base de estas variables, no se considera un gran inconveniente.

Mediante el análisis Anova se evidencia de que las dos variables ("Incremento de la Cohesión familiar", "Invasión del espacio personal”) son significativas para el análisis. Los centros de clústeres finales son los siguientes:

Tabla 3. Centro de clústeres finales-Tipos de relaciones familiares durante la cuarentena

\begin{tabular}{l|c|c|c}
\multicolumn{1}{c}{} & \multicolumn{1}{c}{$\begin{array}{c}\text { Relaciones } \\
\text { conflictivas }\end{array}$} & $\begin{array}{c}\text { Relaciones sin } \\
\text { cambios }\end{array}$ & $\begin{array}{c}\text { Relaciones } \\
\text { fortalecidas }\end{array}$ \\
\hline Cohesión Familiar & $-0,49470$ & $-0,54380$ & 1,20661 \\
\hline $\begin{array}{l}\text { Invasión del } \\
\text { espacio personal }\end{array}$ & 0,95199 & $-0,91285$ & $-0,05924$ \\
\hline
\end{tabular}

Centro de clústeres finales-Tipos de relaciones familiares durante la cuarentena $\mathrm{N}=301$. Pruebas ANOVA para cada variable significativa a un $99 \%$ de confianza

Fuente: Elaboración propia.

Como se mencionó anteriormente, las unidades de medición de las variables analizadas son puntuaciones factoriales. Esto no dificulta, no obstante, la interpretación de los resultados, obteniéndose cuatro grupos claramente diferenciados. Estos han sido nombrados en base al tipo de relación familiar dada durante la cuarentena, pudiéndose clasificar a los encuestados muestra en cuatro grupos según la dinámica de sus relaciones familiares durante la cuarentena.

El clúster "Relaciones conflictivas" hace referencia a aquellos individuos cuyas relaciones familiares han sido afectadas negativamente por la cuarentena, pues no se ha producido un incremento de la cohesión familiar, pero si una invasión del espacio personal. Como dice Juan Carlos Revilla (2020), son “los progenitores que marcan las normas de convivencia” y hay una parte de los jóvenes que vive este aspecto como una imposición y una reducción de la libertad individual, lo cual puede provocar un empeoramiento de "las tensiones derivadas de la convivencia" (Revilla, 2020). Psicólogos, como Lacomba-Trejo et al. (2020), destacan las mayores dificultades de los jóvenes respecto a otros grupos etarios por "la baja resiliencia (...) y las estrategias deficientes de regulación emocional de sus padres". 
Con "Relaciones sin cambios" se recoge aquella población que no considera que la cohesión familiar haya mejorado durante la cuarentena pero que ha mantenido su espacio personal. Este grupo poblacional sería el menos afectado por la cuarentena en lo que a sus relaciones familiares se refiere. Por último, los sujetos integrados en el grupo denominado bajo el nombre "Relaciones fortalecidas" son aquellos cuya cohesión familiar se ha incrementado notablemente durante la cuarentena al mismo tiempo que han mantenido su espacio personal, pudiéndose decir que son la población que ha visto sus relaciones familiares más positivamente afectadas por la cuarentena.

Este análisis deja patente que, detrás la valoración subjetiva de mejora o no mejora de las relaciones familiares (véase el gráfico 1) se esconde una compleja casuística. Si se tiene en cuenta la distribución de la muestra en cada clúster, sólo un 35\% de la población podría decir que no ha experimentado cambios en sus relaciones familiares ("Relaciones sin cambios"), mientras que el resto de la población se divide en similar proporción entre los que han experimentado cambios positivos ("Relaciones fortalecidas": 30\%) y negativos ("Relaciones conflictivas": 35\%).

Esto pone de relieve la pertinencia de utilizar la clasificación de la población en los grupos definidos por el análisis clúster ("Relaciones fortalecidas", Relaciones conflictivas" y "Relaciones sin cambios) para examinar las variaciones de las relaciones familiares durante la cuarentena. De hecho, si se observa tanto la valoración subjetiva del cambio de las relaciones familiares, como el hecho de tener discusiones familiares en relación a los mencionados grupos se obtienen resultados significativos coherentes con las características de los grupos definidos. Los encuestados que tienen "relaciones fortalecidas" son lo que consideran en mayor proporción que sus relaciones familiares han mejorado, al contrario que los que tiene "relaciones conflictivas". Asimismo, la mayoría de los encuestados que tienen "relaciones sin cambios", indican que sus relaciones familiares son iguales que antes.

Tabla 4. Valoración del cambio en las relaciones familiares según el tipo de relación familiar (porcentajes verticales)

\begin{tabular}{c|c|c|c} 
& $\begin{array}{c}\text { Relaciones } \\
\text { conflictivas }\end{array}$ & $\begin{array}{c}\text { Relaciones sin } \\
\text { cambios }\end{array}$ & $\begin{array}{c}\text { Relaciones } \\
\text { fortalecidas }\end{array}$ \\
\hline $\begin{array}{c}\text { Son muchos peores o } \\
\text { peores que antes }\end{array}$ & $35 \%$ & $8 \%$ & $7 \%$ \\
\hline Son iguales que antes & $57 \%$ & $66 \%$ & $46 \%$ \\
\hline $\begin{array}{c}\text { Son mejores o mucho } \\
\text { mejores que antes }\end{array}$ & $8 \%$ & $26 \%$ & $47 \%$ \\
\hline $\mathrm{n}$ & 106 & 104 & 90 \\
\hline \hline
\end{tabular}

Valoración del cambio en las relaciones familiares según el tipo de relación familiar (porcentajes verticales) Chi-cuadrado significativo a un 99\% de confianza

Fuente: Elaboración propia.

Asimismo, aunque en líneas generales la mayoría de la población de estudio ha mantenido discusiones con sus padres durante la cuarentena, el porcentaje de estos casos es mucho menor en "relaciones fortalecidas" y "sin cambios". 
Tabla 5. ¿Ha discutido con sus padres durante la cuarentena? Según tipo de relación familiar (porcentajes verticales)

\begin{tabular}{c|c|c|c|}
\multicolumn{1}{c}{$\begin{array}{c}\text { Relaciones } \\
\text { conflictivas }\end{array}$} & $\begin{array}{c}\text { Relaciones } \\
\text { sin cambios }\end{array}$ & $\begin{array}{c}\text { Relaciones } \\
\text { fortalecidas }\end{array}$ \\
\hline No & $8 \%$ & $45 \%$ & $23 \%$ \\
\hline $\mathrm{Si}$ & $92 \%$ & $55 \%$ & $77 \%$ \\
\hline $\mathrm{n}$ & 106 & 104 & 91 \\
\hline \hline
\end{tabular}

¿Ha discutido con sus padres durante la cuarentena? Según tipo de relación familiar (porcentajes verticales)

Chi-cuadrado significativo a un 99\% de confianza

Fuente: Elaboración propia.

Estos resultados evidencian la pertinencia del modelo obtenido de cara a analizar las preguntas secundarias del presente estudio, relacionadas con el ocio intrafamiliar, las TICs y las posibles consecuencias del confinamiento.

Puede decirse que el ocio intrafamiliar ha aumentado claramente en el grupo "Relaciones Fortalecidas" y en "Relaciones sin cambios" siendo destacable el hecho de que el 25\% de los sujetos integrados en el grupo "Relaciones conflictivas" declare que la frecuencia de ocio ha disminuido.

Tabla 6. Variación en la frecuencia del ocio familiar durante la cuarentena según el tipo de relación familiar (porcentajes horizontales)

\begin{tabular}{c|c|c|c|c}
\multicolumn{2}{c}{ Han disminuido } & $\begin{array}{c}\text { Se han } \\
\text { mantenido igual }\end{array}$ & Han mejorado & $\mathrm{N}$ \\
\hline Relaciones conflictivas & $25 \%$ & $51 \%$ & $24 \%$ & 107 \\
\hline Relaciones sin cambios & $15 \%$ & $44 \%$ & $41 \%$ & 104 \\
\hline Relaciones fortalecidas & $12 \%$ & $33 \%$ & $55 \%$ & 90 \\
\hline
\end{tabular}

Variación en la frecuencia del ocio familiar durante la cuarentena según el tipo de relación familiar (porcentajes horizontales)

Chi-cuadrado sig., al 99\% de confianza.

Fuente: Elaboración propia.

En cuanto al uso de las TICs, se detecta una relación significativa entre el incremento de la frecuencia de uso dispositivos móviles, redes sociales, etc., y el tipo de relaciones familiares durante la cuarentena. Como se puede apreciar en la tabla 7 es la población con "Relaciones fortalecidas" la que declara en menor medida (comparado con el resto de grupos), haber incrementado la frecuencia de uso. No obstante, estos resultados no son concluyentes puesto que el incremento de dispositivos móviles, redes sociales, etc., es generalizado independientemente del tipo de relación familiar. 
Tabla 7. Uso de redes sociales, internet TICs durante la cuarentena según el tipo de relación familiar (porcentajes horizontales)

\begin{tabular}{l|c|c|c}
\multicolumn{2}{c}{$\begin{array}{c}\text { He aumentado la } \\
\text { frecuencia de uso }\end{array}$} & $\begin{array}{c}\text { No he aumentado } \\
\text { la frecuencia de } \\
\text { uso }\end{array}$ & N \\
\hline Relaciones conflictivas & $97 \%$ & $3 \%$ & 105 \\
\hline Relaciones sin cambios & $94 \%$ & $6 \%$ & 105 \\
\hline Relaciones fortalecidas & $89 \%$ & $11 \%$ & 90 \\
\hline
\end{tabular}

Uso de redes sociales, internet TICs durante la cuarentena según el tipo de relación familiar (porcentajes horizontales) Chi-cuadrado sig., al 90\% de confianza.

Fuente: Elaboración propia.

Con estos resultados queda patente que no solo la cohesión familiar es un elemento importante para entender las relaciones familiares, sino que también es necesario atender a otras cuestiones como el uso de las TICs y el respeto por el espacio personal.

\section{Conclusiones y límites}

Los resultados que acabamos de presentar resultan contradictorios, porque hay una parte consistente de los encuestados que declara que no se han producido cambios significativos, a nivel de relaciones familiares, como consecuencia del confinamiento, mientras que otra parte se divide entre quienes hablan de cambios positivos y quienes de cambios negativos. Por otro lado, según nuestro análisis, variables como sexo, edad, tipo de vivienda (metros cuadrados y números de personas con las que se convive) y nivel de ingresos no parecen tener una correlación significativa con la cohesión familiar. Sin embargo, resultan muy interesantes los resultados respecto al ocio intrafamiliar y al uso de las TIC: el ocio intrafamiliar parece influir positivamente sobre la cohesión familiar, mientras que el abuso de las TIC parece influir negativamente.

Por lo que hemos podido averiguar con nuestro estudio, no se puede analizar la cohesión familiar desde el punto de vista de los jóvenes universitarios sin tener en cuenta a la vez el asunto del espacio privado, o personal. En los resultados hemos llegado a clasificar a los encuestados en tres categorías distintas. Y hay un grupo consistente de encuestados, que hemos denominado como grupo de "relaciones conflictivas", en el que la cuarentena parece haber producido efectos negativos sobre el ocio y el diálogo intrafamiliar. Algo que tampoco nos sorprende en exceso, si tenemos en cuenta la edad de los encuestados. Se trata de algo confirmado por otros investigadores, nacionales e internacionales, que han puesto en evidencia la emergencia de conflictos en la organización familiar durante las primeras fases del confinamiento (Vera Vergara, et al., 2020).

A pesar de los objetivos generalizadores de nuestro estudio, no podemos olvidar que cada familia tiene sus propios valores, su propio "idiolecto", utilizando la terminología luhmanniana (Cadenas, 2015: 33). Por consiguiente, tampoco de los porcentajes obtenidos a través de la encuesta podemos deducir automáticamente comportamientos individuales, porque esto podría determinar la llamada "falacia ecológica", un error de atribución muy frecuente en los estudios sociológicos (Martínez Pastor, 2019: 116). 
Un límite de la presente investigación depende del uso exclusivo de técnicas cuantitativas que se ha hecho para recoger los datos. Ya Mora Rojas nos advirtió, en 1994, de las dificultades de estudiar la cohesión familiar solo a través de la encuesta, sin desarrollar técnicas de observación participante. Pero, en el periodo en el que llevamos a cabo la recogida de los datos, los meses de marzo y abril de 2020, resultaba muy complicado la utilización de técnicas cualitativas y la adopción de un enfoque triangular.

No podemos dejar de tener en cuenta que, sobre todo en los países del Sur de Europa, existe una norma social que impone de alguna forma el hablar bien de la propia familia (González y Jurado Guerrero, 2015: 66), aunque, por otro lado, la privatización de la familia y de los lazos familiares ha reducido el peso de la normatividad social. Se trata de algo que puede haber condicionado, por lo menos parcialmente, nuestros resultados.

\section{Referencias}

ARROYO MENÉNDEZ, Millán y FINKEL, Lucila (2019) Encuestas por Internet y nuevos procedimientos muestrales. Panorama Social, (30), 41-53.

BASSETS, Marc. (2020) "Alain Touraine, sociólogo: Esta crisis va a empujar hacia arriba a los cuidadores". El País, 29 de marzo. https://elpais.com/ideas/2020-03-28/alain-touraine-esta-crisis-va-a-empujar-hac ia-arriba-a-los-cuidadores.html Consultado 16 de mayo de 2020.

BAUMAN, Zygmunt (2018) Amor líquido. Barcelona: Paidós.

BECK, Ulrich y BECK-GERNSHEIM, Elisabeth (2003) La individualización. Barcelona: Paidós.

BONILLA, Raquel (2020) Estos son los efectos que provoca el confinamiento en los niños La Razón, https: //www.larazon.es/salud/20200418/6olg4xllvzbrpayqazwcadd4ra.html Consultado el 14 de mayo de 2020.

BYUNG-CHUL Han (2010) La sociedad del cansancio. Barcelona: Herder.

CADENAS, Hugo (2015) La familia como sistema social: conyugalidad y paternalidad. Revista MAD, 33, 29-41.

CALLEJO GALLEGO, Javier (Coords.) (2010) Introducción a las técnicas de investigación social. Madrid: Editorial Universitaria Ramón Areces.

CARABAÑA, Julio (2020) “En favor de las muestras representativas”, El País, 8 de abril. https://elpais.com/so ciedad/2020-04-08/en-favor-de-las-muestras-representativas.html Consultado 12 de mayo de 2020.

CEA D’ANCONA, Ma Ángeles (2002) Análisis multivariable. Teoría y práctica en la investigación social. Madrid: Síntesis.

CEA D’ANCONA, M. (2009) Metodología cuantitativa. Madrid: Síntesis.

CIS (2014) Opiniones y actitudes sobre la familia II. Estudio no 3032. http://www.cis.es. Consultado el 1 de mayo de 2020.

DIRECCIÓN GENERAL DE INNOVACIÓN Y ESTRATEGIA SOCIAL (2020) Informe del Estudio sobre el impacto de situación de confinamiento en la población de la ciudad de Madrid tras la declaración de alarma por la pandemia COVID-19. Impacto económico y laboral sobre los hogares. https://diario.madrid.es/wp-content/uploads/2020/05/Informe-Encuesta-Impacto-Confin amiento-Ciudad-de-Madrid.pdf Consultado el 14 de mayo de 2020.

ERIZALDE SAN MIGUEL, Begoña (2020) La diversidad familiar ante el reto de los cuidados. Dossieres EsF, 36, 34-38.

FUNDACIÓN FOESSA (2019) VIII Informe sobre exclusión y desarrollo social en España. www.foessa.es Consultado el 14 de mayo de 2020. 
Cohesión familiar y covid-19: los efectos de la pandemia sobre las relaciones familiares entre los jóvenes universitarios madrileños y sus

GARCÍA FERRANDO, Manuel; ALVIRA, Francisco., et al. (2015) El análisis de la realidad social. Madrid: Alianza.

GONZÁLEZ, M. ${ }^{\text {a }}$ José y JURADO GUERRERO, Teresa (2015) Padres y madres corresponsables. Madrid: Catarata.

GONZÁLEZ, Rocío (2020) “Mariano Urraco, sociólogo: No creo que el pánico se haya desatado en nuestra sociedad", 21 de marzo https://www.udima.es/es/sociologia-coronavirus-sociedad-mariano-urracoentrevista.html Consultado el 10 de mayo de 2020.

INE (2018) Riesgo de pobreza o exclusión social y de sus componentes por comunidades autónomas, en ht tps://www.ine.es/jaxiT3/Datos.htm?t=10011\#!tabs-tabla Consultado el 14 de mayo de 2020.

KAISER, Henry F. (1974) An index of factorial simplicity. Psychometrika, 39(1), 31-36.

LACOMBA-TREJO, Lauta et al. (2020) Ajuste familiar durante la pandemia de la COVID-19: un estudio de díadas. Revista de Psicología Clínica con Niños y Adolescentes, 7 (3), 66-72.

MARTÍNEZ PASTOR, Juan Ignacio (2019) Los datos sin tapujos. Madrid: Catarata.

MARTUCCELLI, Danilo y SANTIAGO, José (2017) El desafío sociológico hoy. Madrid: CIS.

MEIL, Gerardo (2011) Individualización y solidaridad familiar. Colección Estudios Sociales, número 32. Barcelona: Obra Social La Caixa.

MIELO VIEIRA, Joice y MIRET GAMUNDI, Pau M. (2010) La transición a la vida adulta en España: una comparación en el tiempo y en el territorio utilizando el análisis de entropía. REIS, 131, 75-107.

MILLET, Eva (2020) "La pandemia del Coronavirus pone a prueba la familia", La Vanguardia, 22 de marzo. https://www.lavanguardia.com/magazine/20200322/474261347020/pandemia-coronaviru s-familia-parenting-confinamiento.html Consultado el 15 de mayo de 2020.

MORA ROJAS, Ana Isabel (1994) La dimensión cohesión en la vida familiar. Revista Costaricense de Trabajo Social, 4, 1-12, https://revista.trabajosocial.or.cr/index.php/revista/article/view/158

REHER, David (1996) La familia en España. Pasado y presente. Madrid: Alianza Editorial.

REVILLA, Juan Carlos (2020) ¿Por qué las personas jóvenes podrían estar viviendo peor el confinamiento? Una explicación sociológica. Análisis y Debate. Blog del Centro Reina Sofía sobre Adolescencia y Juventud, https://www.adolescenciayjuventud.org/blog/analisis-y-debate/por-que-las-personas-jov enes-podrian-estar-viviendo-peor-el-confinamiento-una-explicacion-sociologica/

ROLLAND, John S. (2012) "Mastering family challenges in serious illness and disability", en WALSH, Froma (Ed.). Normal family processes. New York: Guilford Press, pp. 452-482.

SANTORO, Pablo (2020). Coronavirus: la sociedad frente al espejo. https://ethic.es/2020/03/sociologia-de l-coronavirus-la-sociedad-frente-al-espejo/ Consultado el 15 de mayo de 2020.

SEGALEN, Martine (2013) Sociología de la familia. Argentina: EUDEM.

SZOLNOKI, Gergely y HOFFMANN, Dieter (2013) Online, face-to-face and telephone surveys-Comparing different sampling methods in wine consumer research. Wine Economics and Policy, 2(2), 57-66, http s://doi.org/10.1016/j.wep.2013.10.001

TARDIVO, Giuliano (2016) Aproximación a la Sociología contemporánea. Barcelona: UOC.

VERA VERGARA, Vivan et al. (2020) Funcionamiento familiar durante la pandemia: experiencias desde el psicogrupo adultos vs. Covid-19. Revista Cubana de Psicología, 2 (2), 41-50.

YI-LING, Liu (2020) Is Covid-19 changing our relationships?, en BBC-Future, 5 junio. https://www.bbc.com /future/article/20200601-how-is-covid-19-is-affecting-relationships Consultado el 15 julio 2020 
Tardivo, Suárez-Vergne, Díaz Cano

Notas

1 Es importante destacar que lo que se mide con este componente es el Incremento vs No incremento de la cohesión familiar durante la cuarentena no el grado de cohesión familiar en sí mismo 